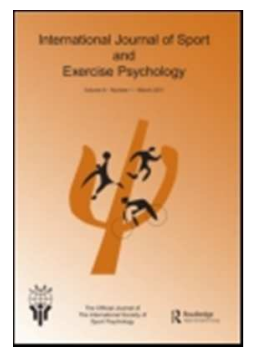

\title{
A distal focus of attention leads to superior performance on a golf putting task
}

\begin{tabular}{|r|l|}
\hline Journal: & International Journal of Sport and Exercise Psychology \\
\hline Manuscript ID: & RIJS-2014-0080.R1 \\
\hline Manuscript Type: & Original Article \\
\hline Keywords: & attentional focus, motor learning, golf \\
\hline \multicolumn{2}{|}{} \\
\end{tabular}

SCHOLARONE ${ }^{m}$

Manuscripts 


\title{
A.A. Author
}

\section{A distal focus of attention leads to superior performance on a golf putting task}

\begin{abstract}
The purpose of this experiment was to determine the optimal focus of attention for novice golfers performing a putting task. Previous research has advocated that novices should adopt a proximal external focus for novices, but this advice has been drawn from research on a relatively complex task (i.e., pitch shot). Research examining golf putting specifically has failed to find an advantage of adopting either a proximal or an internal focus, but experiments has have not included a distal focus condition. The present research investigated if adopting a distal external focus of attention would lead to superior putting performance in novices. Following familiarization with the task, general putting technique, and the concept of attentional focus, 18 participants completed three sets of 15 putts in a counter-balanced, within participant design, adopting a different focus of attention for each set (internal, proximal external, distal external). After every five putts, participants were asked to answer three questions concerning how much they focused on internal, proximal or distal cues. On the completion of the trials, participants were asked to identify if they had a preference for one or other focus. The self-reports indicated that participants adhered to the three instructional conditions. Performance in the distal focus condition was significantly better than performance in the proximal or internal conditions, which did not differ. Significantly more participants preferred a distal focus of attention than would be expected by chance. Task complexity appears to be an important variable in the selection of the optimal external focus of attention for novices.
\end{abstract}

Keywords: attentional focus; motor learning; golf

Research into attentional focus has led to recommendations for how to optimally phrase instructions to enhance practice quality. In this study, attentional focus refers to the specific thought adopted by a performer during the execution of a motor skill. Building from the initial investigations by Wulf and colleagues (Wulf, Höß, \& Prinz, 1998), two primary categories of attentional focus have been investigated. An internal focus refers 
to attention directed towards specific body movements. For example, a golfer narrowing his/her thoughts to maintaining the hinge in the wrists through impact in a pitch shot (e.g., Bell \& Hardy, 2009) would be adopting an internal focus. In contrast, an external focus refers to focusing on the effect of one's body movements. To continue the golfing example, directing attention to the pendulum-like motion of the club (e.g., Wulf \& Su, 2007) would be an example of an external focus. Research has consistently demonstrated an advantage for external focus instructions relative to internal or control (i.e., no instruction) conditions in a wide variety of tasks (for reviews see Wulf, 2007; Lohse, Wulf, \& Lewthwaite, 2012).

The dominant explanation for the benefits of an external focus of attention is provided by the constrained action hypothesis (McNevin, Wulf, \& Shea, 2003; Wulf, McNevin, \& Shea, 2001). According to this hypothesis, an internal focus interferes with automatic control processes that would otherwise produce the movement more efficiently. Evidence in support of the constrained action hypothesis has been provided by findings that external focus conditions have resulted in reduced secondary task reaction time (Wulf et al., 2001), higher frequency movement adjustments (McNevin et al., 2003; Wulf et al., 2001), and more efficient EMG profiles (Vance, Wulf, McNevin, Töllner, \& Mercer, 2004).

Golf provides a range of variables on which attention could be focused. In addition to internal factors, golfers could attend to proximal (that is, close to the body) external factors such as the club motion, or more distal effects (that is, further from the body) such as the desired trajectory of the ball (Bell \& Hardy, 2009; Wulf, McNevin, Fuchs, Ritter, \& Toole, 2000). Wulf and Su (2007) hypothesised that beginners' performance may be enhanced by initially directing focus to proximal effects, such as club motion. As skill level is improved, the optimal focus is proposed to become 


\section{A.A. Author}

progressively more distal. Early research into focus of attention in golf is supportive of this hypothesis. Wulf, Lauterbach, and Toole (1999; see also Wulf and Su, 2007, experiment one) demonstrated that, for novices, a proximal external focus of attention led to an immediate advantage during practice of a pitch shot relative to an internal focus. Furthermore, this advantage was sustained on a retention test conducted the next day. Wulf et al. (2000, experiment two) had novice participants practice the same pitching task as Wulf et al. (1999). Participants assigned to a proximal condition focused on the motion of the club. Participants assigned to a distal condition focused on the desired trajectory and the target. The proximal focus was found to produce an immediate advantage during practice, and this advantage was again sustained on a retention test conducted the next day. The authors suggested that, given the complexity of the pitching task, the novices could not relate changes in distal outcome to changes in the underlying movement pattern. Focusing on controlling the golf club provided more salient information about their movements. Thus it appears that, for the relatively complex pitch shot, a proximal focus of attention leads to enhanced performance in novices.

Perkins-Ceccato, Passmore, and Lee (2003) extended research on focus of attention in golf to examine if skill level influenced the effectiveness of an internal or external focus. As the previous studies would suggest, the high skill golfers performed more consistently when they were provided with an external focus. However, the low skill golfers performed with less consistency when provided with an external focus. Although initially appearing to contradict the results of Wulf and colleagues, the design of Perkins-Ceccato et al. (2003) has been criticised with respect to the instructions which were provided to participants. In the internal condition, participants were asked to focus on the form of the golf swing, and to adjust the force of their swing depending 
on the distance of the shot. This instruction does not prevent participants from focusing on the motion of the club: that is, adopting a proximal external focus. In the external condition, participants were encouraged to concentrate on hitting the ball as close to the target as possible. This instruction may be classified as a distal external focus.

Therefore another possible explanation of Perkins-Ceccato et al.'s (2003) result is that the specific external focus was too far removed from the body movements responsible to be relevant to the novice performer (Wulf \& Prinz, 2001). The absence of some form of manipulation check (see Marchant, Clough, Crawshaw, \& Levy, 2009) is an important limitation which complicates interpretation of Perkins-Ceccato et al.'s results.

Bell and Hardy (2009) addressed some of the issues raised by Perkins-Ceccato et al. (2003) by examining the effect of three attentional foci (internal, external proximal and external distal) on the golf pitch shot performance of skilled golfers (mean handicap 5.51). A manipulation check was included which indicated that participants successfully directed their attention as instructed. The distal external focus group performed significantly more accurately than a proximal external group and an internal group, while the proximal external group also performed more accurately than the internal group. Thus, when examined in the relatively complex task of pitching, there appears to be support for Wulf and Su's (2007) suggested progression from proximal to distal focus of attention with an increase in skill level.

A second hypothesis postulated by Wulf and $\mathrm{Su}$ (2007) was that actions should always be controlled at the most distal level possible. Although no less difficult, putting is a less complex motor task than pitching when considered in terms of the number of active joints, and the independence and range of motion of the joint actions. Due to the lower complexity of putting, a distal focus may prove effective for novices on this task. Relatively little research has focused on the impact of attentional focus instructions on 
putting performance. One exception to this is a study by Poolton, Maxwell, Masters, and Raab (2006). An internal group was directed to focus on the swing of their hands, while an external group was directed to focus on the swing of the club head. No significant differences were found for putting performance in the practice or retention phases, although under secondary task loading the internal group suffered a greater decrement in performance. One possible reason for the lack of differences between groups may be the sensitivity of the measure used: the number of putts holed. Although putting from a relatively short distance, the groups' best performances saw less than $33 \%$ of attempts scored. This lack of sensitivity in the measure may be one reason why no differences between the groups emerged.

A second study on putting by Tranter (as cited in Wulf, 2007) also demonstrated no significant differences between internal (focus on the movement of the hands) and external (focus on the movement of the club) groups. Wulf (2007) interpreted this finding as support for the notion that a degree of difficulty is required for the focus of attention effect to appear. This position is supported by Poolton et al.'s (2006) finding that differences between the internal and external groups only emerged on the most difficult secondary task condition. However, an alternative explanation for the results of the studies by Tranter and by Poolton et al. is that an overly proximal focus was used in both cases. Following Wulf and Su's (2007) recommendation that actions should be controlled at the most distal level, it is possible that directing attention to a more distal effect, such as desired ball trajectory, would lead to superior putting performance relative to an internal or proximal external condition. Support for this proposition comes from McKay and Wulf (2012) who demonstrated an advantage for a distal relative to a proximal external focus of attention in novices on a dart throwing task. Due to the use of an implement to strike an object in golf putting, rather than direct 
manipulation of the object as in darts, putting may be classified as intermediate between dart throwing and pitching on a continuum from simple to complex movements.

Therefore, the results of McKay and Wulf (2012) may not generalise to a putting task.

An alternative explanation for the null effects demonstrated by the Tranter (as cited in Wulf, 2007) and Poolton et al. (2006) experiments on putting additional eonsideration isrelates to the effect of individual preference for internal or external focus of attention instructions. Wulf, Shea, and Park (2001) initially found no support for individual preferences influencing instructional effectiveness in a balance task; while the majority of participants preferred an external focus, adopting an external focus of attention resulted in superior performance irrespective of preference. However, subsequent research by Marchant et al. (2009) using a dart throwing task, and by Weiss, Reber, and Owen (2008) using basketball shooting, has suggested that individual preference may influence the effectiveness of attentional focus instructions. Adopting an internal focus was found to negatively impact individuals with a preference for an external focus, however, adopting an external focus had no effect on the performance of participants who preferred an internal focus. Althouhgh unlikely, It it is possible that individual preference is responsible for the nulleffects demonstrated in thethe majority of participants in the experiments by Tranter (as cited in Wulf, 2007) and Poolton et al. (2006) experiments on putting had a preference for adopting an internal focus. As such, an attempt to identify the optimal attentional focus for putting should measure $\underline{\text { report }}$ individual-participant preferences as a potential confounding variable.

A key concern in the focus of attention literature is the degree to which participants adhere to instructions. Early research (e.g., Wulf, et al., 1998) has been criticised for the absence of manipulation checks. Subsequent research has utilised a number of different methods of self-report, including categorical questions (e.g., did 
you focus as instructed; Freudenheim, Wulf, Madureira, Pasetto, \& Corrêa, 2010; Stoate \& Wulf, 2011), open questions (e.g., what did you focus on, Stoate \& Wulf, 2011; Porter, Nolan, Ostrowski, \& Wulf, 2010) and closed questionnaires (e.g., how much did you focus on x, Bell \& Hardy, 2009; Marchant et al., 2009). Checks have been administered both during (e.g., Marchant et al., 2009; Porter et al., 2010) and post experiment (e.g., Freudenheim et al., 2010; Stoate \& Wulf, 2010). There is clearly a lack of standardization in the administration of manipulation checks. Within the literature on associative/dissociative focus of attention, the use of both Likert-type manipulation checks during the activity, and open questions post activity, is recommended (Tenenbaum \& Connolly, 2008; Masters \& Ogles, 1998). Investigations of internal/external focus of attention would be strengthened by the inclusion of multiple manipulation checks.

Regardless of the type of manipulation check implemented, there remains a concern with social desirability bias (SDB) when using self-report measures. SDB refers to participants responding to a questionnaire or experimenter in a manner which they believe the experimenter will be pleased with (Fisher, 2000). Questionnaire studies frequently include an additional scale to assess SDB (for an example in a sporting context, see Gucciardi, Gordon, \& Dimmock, 2009). A further enhancement of manipulation checks within the focus of attention literature may be to include an assessment of SDB (e.g., Reynolds, 1982) to increase confidence in the validity of the self-reports.

The purpose of the current study was to investigate Wulf and Su's (2007) proposal that action should always be controlled at the most distal level possible. Previous research has identified that a proximal external focus is optimal for novice golfers on relatively complex pitching tasks. No optimal focus has been identified for 
the less complex task of putting. Crucially, previous experiments have only compared proximal and internal foci. The present study examined whether a more distal focus would promote superior putting performance in novice golfers. Individual preferences for differing attentional focus instructions were measured as a potential confounding variable, and a rigorous set of manipulation checks were applied to ensure participants were focusing as directed. Due to the low complexity of the skill, it was hypothesised that the performance of novices would be highest in a distal external focus condition, and that more participants would prefer a distal external focus of attention. Based on the findings of previous research on putting, it was also hypothesised that performance would not differ between proximal external and internal focus conditions.

\section{Method}

\section{Participants}

Eighteen volunteers ( 4 women and 14 men) between the ages of 18 and $26(M$ age $=$ 20.7 years) participated in this study. Participants did not play any form of golf (i.e., pitch \& putt, miniature golf) on more than three occasions per year. No participant had any experience of competitive golf. Informed consent was obtained from all participants, and ethical approval was obtained from the University Research Ethics Committee.

\section{Apparatus and Task}

Participants were required to putt on an indoor putting surface towards a target $4 \mathrm{~m}$ distant from each of five locations. The target consisted of a series of concentric circles and participants were asked to stop the golf ball in the centre circle (diameter $10 \mathrm{~cm}$ ). Putts that went off the matt could not be accurately measured, therefore the distance of 


\section{A.A. Author}

each putt from the target was converted into points, with 15 points awarded for stopping the ball within $5 \mathrm{~cm}$ of the centre of the target, 14 points for a ball within $10 \mathrm{~cm}$, and so on to 0 points.

\section{Procedure}

Consistent with previous research on focus of attention in novice golfers (Wulf et al., 1999), during an initial familiarization phase, participants were provided with a basic guide to putting: (1) adopt a comfortable position, (2) look at the target, (3) look at the ball, and (4) play the stroke. Pilot testing revealed that this basic guide increased the consistency of participants' initial putting performances. Participants then performed 10 putts with no focus instructions to familiarize themselves with the task. At this point participants were introduced to the concept of focusing attention. Specifically, focus of attention was defined as the particular thought that a performer deliberately adopted during the execution of a skill. Immediately before and during execution (i.e., during steps 3 and 4 of the basic guide to putting), participants were to instructed to narrow their focus down to just the one thought that they were given and to block out other thoughts as best they could.

During pilot testing, no one instruction emerged as most appropriate for each category of focus. As such, participants did not receive a single instructed focus, but an explanation of the category of focus, and a number of examples that they could potentially use. Participants were instructed to adopt one focus that was consistent with the definition of the category. The internal (body) focus was defined as thinking about the specific body movements necessary to leave the ball on the target, such as the distance the arms move backwards and forwards, or the smooth swing required from the shoulders. The proximal external (club) focus was defined as thinking about what you 
need the club to do to leave the ball on the target; for example, the distance the club head moves backwards and forwards, or a smooth pendulum like swing of the club. The distal external (ball path) focus was defined as thinking about what you want to happen, and holding an image of that desired outcome in your mind as you execute the skill. The examples provided included drawing an imaginary line from the ball to the centre of the target, or imaging the final position of the ball during the stroke.

For each condition, participants initially received a block of five trials to familiarize themselves with the focusing instructions. At any point during the explanation and the five familiarization putts, participants were encouraged to ask questions to clarify their understanding of focusing attention, or of the one specific focus that they were using. Following the familiarization with the focusing instructions, participants completed two blocks of five putts which were measured. Participants completed all three conditions (internal, proximal external, and distal external focus) in a counterbalanced order. Between each set of five putts, participants were asked a series of questions to which they responded using a visual analogue scale from 0 (not at all) to 4 (very much so): (1) to what extent were you focusing on the movement of any part of your body? (2) to what extent were you focusing on the movement of your club? (3) to what extent were you focusing on the ball path? (4) how difficult was it to narrow your focus down to just the instructed focus? (5) was there anything else that you were thinking about during the execution of the stroke? Participants were reminded of their focus before each set of five shots. Between conditions, participants received one minutes rest before the next condition was explained.

To increase confidence in the manipulation checks, upon completion of the three conditions, participants were asked to describe precisely what they were focusing on in each condition. Participants were then asked to indicate which, if any, of the three 


\begin{abstract}
A.A. Author
conditions they preferred. Finally, to address the risk of social desirability bias influencing the self-report scores, participants completed Reynolds' (1982) 13-item short form Marlowe-Crowne Social Desirability Scale. Participants respond with either True or False to indicate their agreement with 13 socially desirable statements (e.g., I'm always willing to admit it when I make a mistake). The short form has been reported as having adequate internal consistency $\left(\mathrm{r}_{\mathrm{KR}-20}=0.76\right)$ and correlation with the original 33 item scale $(\mathrm{r}=0.93)$ (Reynolds, 1982). Participants were excluded from the data set if they reported a median focus less than two on the instructed focus for any condition, or if they reported a median focus for an uninstructed focus direction equal to or exceeding that reported for the instructed focus.
\end{abstract}

\title{
Data Analysis
}

The median responses to the self-report manipulation check questions in each of the three conditions was compared using Friedman's ANOVA by ranks, with follow up Wilcoxon signed ranks tests as necessary.

Friedman's ANOVA by ranks was also used to analyse the median putting performance, and the question on the perceived difficulty of the three conditions. A chi-squared goodness of fit test was used to analyse differences in the number of participants who reported a preference for each condition. For each analysis the alpha level was set as 0.05 .

\section{Results}

In terms of the attentional focus manipulation, all 18 participants reported focusing as directed by the instructions (see figure 1). For example, in the internal focus condition, a participant would be expected to report higher focus on body movements compared to 
their focus on the club or on the ball path. Friedman's ANOVAs revealed that within each condition, the instructed focus was rated significantly higher than the uninstructed foci: internal, $\chi^{2}(2, N=18)=29.939, p<0.001 ;$ proximal, $\chi^{2}(2, N=18)=29.059, p<$ 0.001 ; distal, $\chi^{2}(2, \mathrm{~N}=18)=27.114, \mathrm{p}<0.001$. Follow up Wilcoxon signed ranks tests with Bonferroni-Holm corrections revealed that, in each case, the instructed focus was rated significantly higher than the other foci which did not differ. The open questions were coded by an independent rater who was blind to the conditions. The answers supported the results of the self-report questions; for example participant 18 reported for internal focus: "arms still and controlled, no elbow bend", for proximal focus: "keep club controlled in backswing, not rushed", and for distal focus: "imagine line and trying to get the ball along the line". Controlling for an additional potential confounding variable, Friedman's ANOVA revealed no significant difference between the conditions in the ratings of how difficult it was to follow the instructions (internal, $M d n=2$; proximal, $M d n=2 ;$ distal, $M d n=1.75), \chi^{2}(2, \mathrm{~N}=18)=2.59, \mathrm{p}=0.274$.

Following the recommendation of Edens, Buffington, Tomicic, and Riley (2001), where a score on the social desirability scale in excess of 10 was reported the self-report manipulation check data, and the answers to the open questions were reexamined. Scores on the social desirability scale ranged from 4-10, with a median of 7 . Based on the agreement of the answers to the open questions with the desired categories, and the variation in scores provided on the self-report manipulation check questions, no data was excluded.

Friedman's ANOVA by ranks revealed significant differences between the number of points accumulated (higher is better) in the internal (Median $=3.75)$, proximal $($ Median $=3.6)$ and distal $($ Median $=4.85)$ conditions, $\chi^{2}(2, \mathrm{~N}=18)=8.48, \mathrm{p}=$ 0.014 (figure 2). Follow-up pairwise comparisons conducted using Wilcoxon signed 
ranks tests with Bonferroni-Holm corrections revealed that performance in the distal condition was significantly higher than performance in the internal, $T=-0.861, \mathrm{p}=$ $0.029, \mathrm{r}=-0.57 ;$ and proximal conditions, $T=-0.806, \mathrm{p}=0.047, \mathrm{r}=-0.61$. The internal and proximal conditions did not differ $(\mathrm{p}>0.05)$.

A chi-squared goodness of fit test revealed that preference for the three conditions was not equally distributed, $\chi^{2}(2, \mathrm{~N}=18)=6.33, \mathrm{p}=0.042$, Cramer's $\mathrm{V}=$ 0.593. More participants preferred a distal $(n=11)$ relative to a proximal $(n=3)$ or an internal $(n=4)$ focus of attention than would be expected by chance.

\section{Discussion}

The purpose of this experiment was to determine the optimal focus of attention for novice golfers performing a putting task. Previous research has advocated a proximal external focus for novices (Wulf et al., 2000; Wulf \& Su, 2007), but this advice has been drawn from research on a relatively complex task (the pitch shot). In the present experiment, a distal focus was found to be most effective for the relatively low complexity task of putting. This finding adds to the literature by indicating that optimal attentional focus depends not just on performer skill level, but also on the complexity of the skill being performed. More specifically, the results explain that the failure of previous research on putting to identify an effect of attentional focus instructions (Poolton et al., 2006; Tranter, as cited in Wulf, 2007) may have been due to the limited range of instructions investigated (i.e., proximal external and internal foci only). In addition, this finding supports the recommendation of Wulf and $\mathrm{Su}$ (2007) that an action should always be controlled at the most distal level possible.

It is not clear why no difference emerged between the proximal external and internal conditions. Previous research with novice golfers performing a complex 
pitching task have demonstrated an advantage for a proximal focus over an internal focus (Wulf et al., 2000; Wulf \& Su, 2007). However, for putting, research by Tranter (as cited in Wulf, 2007) and by Poolton et al. (2006) has consistently demonstrated no significant difference between proximal external and internal focus conditions. One possible explanation is that with more complex tasks it is easier to distinguish between body movements and the proximal effects that they generate (Wulf, 2007). In contrast, with less complex tasks such as putting, such a distinction may be more difficult to make. An alternative explanation is that due to its relative simplicity, participants may be able to effectively focus on remote effects within the golf putt. As such, adopting a proximal external focus may interfere with automatic control processes similar to the adoption of an internal focus.

Action Identification Theory (AIT, Vallacher \& Wegner, 1987, 2012) offers support for this latter explanation for the similarity in performance of the internal and proximal groups. The theory proposes that a performer's thoughts during execution will be drawn to one of a hierarchy of goal relevant mental representations known as act identities. For example, a golfer standing over a putt may think about "winning the tournament", "sinking the putt", "sending the ball along the path to the hole", "forward and back movement of the club", or the specific mechanics of the actionbody movements required. The former examples represent high level act identities, with the level decreasing through the examples. According to AIT, the optimal level of act identity to adopt is dependent upon a range of factors, including task difficulty and individual skill level. Adopting too high or too low an act identity, whether through instructions, lack of concentration or as a response to anxiety, is predicted to lead to suboptimal performance. Support for the proposition that an external focus may in certain circumstances represent an inappropriately low level of act identity comes from 
Wulf (2008) in which elite (Cirque du Soleil) balance acrobats suffered performance decrements under both internal and external focus conditions, in a balance task which had previously demonstrated a performance advantage for an external focus in adult novices (Wulf, Töllner, \& Shea, 2007, experiment two). Thus, due to the relatively low complexity of the skill of putting, it is possible that a proximal focus is an inappropriately low level act identity for the skill of putting, even in novices.

The skill level of the individual and the complexity of the task have been shown to be important factors to consider when applying core principles of skill acquisition, such as contextual interference and feedback frequency, to practice design (Guadagnoli $\&$ Lee, 2004). Given that optimal focus of attentional appears to be similarly sensitive to both skill level and task demands, it is important for sport psychologists and coaches to understand the nature of focus of attention as a continuum (Wulf \& Su, 2007). Practical guidelines might include identifying a range of possible proximal and distal external foci for each task, and encouraging the learner to adopt the most distal focus that he or she believes can be effectively controlled. Consistent with previous research (McKay \& Wulf, 2012), participants in the current study reported a preference for a distal focus of attention. Preference has been demonstrated to have an influence on the effectiveness of focus of attention instructions (Marchant et al., 2009; Weiss et al., 2008), hence preference is an important potential confounding variable to measure. Future research should consider how learner preference changes over time on a task, and the appropriateness of using learner preference to dictate the timing of shifts from proximal to distal attentional focus over the course of learning a complex task.

$\underline{\text { One potential limitation of the reported experiment comes from the instructions }}+$ provided to the participants. The attentional focus during the execution of the skill was $\underline{\text { carefully controlled and monitored. However, as they were preparing their stance, }}$ 
participants were free to focus as they chose. The rationale for this decision stemmed from Wulf's (2007) specification that the critical time to control attentional focus is during the execution of the movement. This decision is also supported by the fact that both generic (e.g., Singer, 1988) and golf-specific learning strategies (e.g., Lee \& Schmidt, 2014) emphasise the need to consider separately the thoughts before and those during the execution of sport skills; a point echoed by popular golf instruction texts (e.g., Nicklaus \& Bowden, 1974; Montgomerie, 2003). Nonetheless, the lack of consistency in participants preparatory thoughts is a potential confounding variable.

A second limitation is that task complexity was not manipulated directly. Instead, this paper extrapolates from the findings of research investigating similar and more complex golfing tasks which may have followed different protocols to the present experiment (Bell \& Hardy, 2009; Poolton et al., 2006; Wulf et al., 2000; Wulf \& Su, 2007). Golf is ideally suited to the examination of the effect of complexity on optimal attentional focus, given the gradual increase in active joints, the range of motion in the active joints, and therefore the demand on coordination, as the distance of the ball from the target increases. Future research should attempt to confirm the proposed impact of task complexity on optimal attentional focus through a direct manipulation of task complexity.

Formatted: Font: $12 \mathrm{pt}$

A key concern in the focus of attention literature is the degree to which participants adhere to instructions. Previous research has indicated that the use of instructions is less than 100\% (Marchant, Clough, \& Crawshaw, 2007; Marchant et al., 2009), or that participants do not always focus exclusively internally or externally (Porter et al., 2010). Guided by recommendations from the associative/dissociative focus of attention literature (Masters \& Ogles, 1998), the present study utilised Likerttype manipulation checks during breaks in the activity, open questions post activity, and 


\section{A.A. Author}

a measure of social desirability bias (SDB). Although no data was excluded in the present study, the use of multiple self-report measures and the SDB questionnaire gave increased confidence that the participants were focusing as directed. Future research should address the appropriateness of using the SDB questionnaire outside of the survey environment for which it was designed. In addition, the use of a think aloud protocol (e.g., Toner \& Moran, 2011) as an alternative to Likert-type probes could be explored. Research on focus of attention would benefit from a review suggesting clear guidelines for the use of manipulation checks, and criteria for the exclusion of participants.

In conclusion, it appears that the complexity of the task influences the optimal focus of attention. In contrast to findings with more complex pitching tasks, previous research in golf putting had found no benefit to adopting either a proximal or an internal focus of attention. The current study extended previous research by including an additional distal external focus of attention condition. In a novice population, the distal external focus of attention led to superior putting performance relative to a proximal external or internal focus. As such, this study supports Wulf and Su's (2007) suggestion that action should be controlled at the most distal level possible. 
International Journal of Sport \& Exercise Psychology

\section{References}

Bell, J. J., \& Hardy, J. (2009). Effects of attentional focus on skilled performance in golf. Journal of Applied Sport Psychology, 21, 163-177. doi: $10.1080 / 1041320090279532$.

Edens, J. F., Buffington, J. K., Tomicic, T. L., \& Riley, B. D. (2001). Effects of positive impression management on the Psychopathic Personality Inventory. Law and Human Behavior, 25, 235-256. doi: 10.1023/a:1010793810896.

Fisher, R. J. (2000). The future of social desirability research in marketing. Psychology and Marketing, 17, 73-77. doi: 10.1002/(SICI)1520-6793(200002)17:2<73::AIDMAR1>3.0.CO;2-L.

Freudenheim, A. M., Wulf, G., Madureira, F., Pasetto, S. C., \& Correa, U. C. (2010). An external focus of attention results in greater swimming speed. International Journal of Sports Science \& Coaching, 5, 533-542. doi: 10.1260/17479541.5.4.533.

Guadagnoli, M. A., \& Lee, T. D. (2004). Challenge point: A framework for conceptualizing the effects of various practice conditions in motor learning. Journal of Motor Behavior, 36, 212-224. doi: 10.3200/JMBR.36.2.212-224.

Gucciardi, D. F., Gordon, S., \& Dimmock, J. A. (2009). Development and preliminary validation of a mental toughness inventory for Australian football. Psychology of Sport \& Exercise, 10, 201-209. doi: 10.1016/j.psychsport.2008.07.011.

Lee, T. D., \& Schmidt, R. A. (2014). PaR (Plan-act-Review) golf: Motor learning research and improving golf skills. International Journal of Golf Science, 3, 2-25. doi:10.1123/ijgs.2014-0004 
Lohse, K. R., Wulf, G., \& Lewthwaite, R. (2012). Attentional focus affects movement efficiency. In N. J. Hodges \& A. M. Williams (Eds.), Skill Acquisition in Sport: Research, Theory and Practice (2nd ed., pp. 40-58). London: Routledge.

Marchant, D. C., Clough, P. J., \& Crawshaw, M. (2007). The effects of attentional focusing strategies on novice dart throwing performance and their task experiences. International Journal of Sport and Exercise Psychology, 5, 291-303. doi: 10.1080/1612197X.2007.9671837.

Marchant, D. C., Clough, P. J., Crawshaw, M., \& Levy, A. (2009). Novice motor skill performance and task experience is influenced by attentional focusing instructions and instruction preferences. International Journal of Sport and Exercise Psychology, 7, 448-502. doi: 10.1080/1612197X.2009.9671921.

Masters, K. S. \& Ogles, B. M. (1998). Associative and dissociative cognitive strategies in exercise and running: 20 years later, what do we know? The Sport Psychologist, 12, 253-270. Retrieved from http://journals.humankinetics.com/tsp.

McKay, B., \& Wulf, G. (2012). A distal external focus enhances novice dart throwing performance. International Journal of Sport and Exercise Psychology, 10, 149156. doi: 10.1080/1612197x.2012.682356.

McNevin, N. H., Shea, C. H., \& Wulf, G. (2003). Increasing the distance of an external focus of attention enhances learning. Psychological Research, 67, 22-29. doi: 10.1007/s00426-002-0093-6.

Montgomerie, C. (2003). The thinking man's guide to golf. London: Orion.

Nicklaus, J., \& Bowden, K. (1974). Golf my way. London: Heinemann.

Perkins-Ceccato, N., Passmore, S. R., \& Lee, T. D. (2003). Effects of focus of attention depend on golfers' skill. Journal of Sports Sciences, 21, 593-600. doi:

10.1080/0264041031000101980. 
Poolton, J. M., Maxwell, J. P., Masters, R. S. W., \& Raab, M. (2006). Benefits of an external focus of attention: Common coding or conscious processing? Journal of Sports Sciences, 24, 89-99. doi: 10.1080/02640410500130854.

Porter, J. M., Nolan, R. P., Ostrowski, E. J., \& Wulf, G. (2010). Directing attention externally enhances agility performance: a qualitative and quantitative analysis of the efficacy of using verbal instructions to focus attention. Frontiers in Psychology, 1,216. doi: 10.3389/fpsyg.2010.00216.

Reynolds, W. M. (1982). Development of reliable and valid short forms of the Marlowe-Crowne Social Desirability Scale. Journal of Clinical Psychology, 38, 119-125. doi: 10.1002/1097-4679(198201)38:1<119::aidjclp2270380118>3.0.co;2-i.

Singer, R. N. (1988). Strategies and metastrategies in learning and performing selfpaced tasks. The Sport Psychologist, 2, 49-68. Retrieved from http://journals.humankinetics.com/tsp.

Stoate, I., \& Wulf, G. (2011). Does the attentional focus adopted by swimmers affect their performance? International Journal of Sports Science \& Coaching, 6, 99-108. doi: 10.1260/1747-9541.6.1.99.

Tenenbaum, G., \& Connolly, C. T. (2008). Attention allocation under varied workload and effort perception in rowers. Psychology of Sport \& Exercise, 9, 704-717. doi: 10.1016/j.psychsport.2007.09.002.

Toner, J. T., J., \& Moran, A. (2011). The effects of conscious processing on golf putting proficiency and kinematics. Journal of Sports Sciences, 29, 673-683. doi: $10.1080 / 02640414.2011 .553964$. 


\section{A.A. Author}

Vallacher, R. R., \& Wegner, D. M. (1987). What do people think they’re doing - action identification and human behavior. Psychological Review, 94, 3-15. doi: 10.1037//0033-295x.94.1.3.

Vallacher, R. R., \& Wegner, D. M. (2012). Action identification theory: The highs and lows of personal agency. In P. Van Lange, A. W. Kruglanski \& E. T. Higgins (Eds.), Handbook of theories in social psychology (pp. 327-348). London: Sage.

Vance, J., Wulf, G., Tollner, T., McNevin, N., \& Mercer, J. (2004). EMG activity as a function of the performer's focus of attention. Journal of Motor Behavior, 36, 450459. doi: 10.3200/JMBR.36.4.450-459.

Weiss, S. M., Reber, A. S., \& Owen, D. R. (2008). The locus of focus: The effect of switching from a preferred to a non-preferred focus of attention. Journal of Sports Sciences, 26, 1049-1057. doi: 10.1080/02640410802098874.

Wulf, G. (2007). Attention and Motor Skill Learning. Champaign, IL: Human Kinetics. Wulf, G. (2008). Attentional focus effects in balance acrobats. Research Quarterly for Exercise \& Sport, 79, 319-325. doi: 10.1080/02701367.2008.10599495.

Wulf, G., Hob, M., \& Prinz, W. (1998). Instructions for motor learning: Differential effects of internal versus external focus of attention. Journal of Motor Behavior, 30, 169-179. doi: 10.1080/00222899809601334.

Wulf, G., Lauterbach, B., \& Toole, T. (1999). The learning advantages of an external focus of attention in golf. Research Quarterly for Exercise and Sport, 70, 120-126. doi: 10.1080/02701367.1999.10608029.

Wulf, G., McNevin, N. H., Fuchs, T., Ritter, F., \& Toole, T. (2000). Attentional focus in complex skill learning. Research Quarterly for Exercise and Sport, 71, 229-239. doi: 10.1080/02701367.2000.10608903. 
Wulf, G., McNevin, N., \& Shea, C. H. (2001). The automaticity of complex motor skill learning as a function of attentional focus. Quarterly Journal of Experimental Psychology Section A - Human Experimental Psychology, 54, 1143-1154. doi: $10.1080 / 713756012$.

Wulf, G., \& Prinz, W. (2001). Directing attention to movement effects enhances learning: a review. Psychonomic Bulletin \& Review, 8, 648-660. doi: 10.3758/BF03196201.

Wulf, G., Shea, C., \& Park, J. H. (2001). Attention and motor performance: Preferences for and advantages of an external focus. Research Quarterly for Exercise \& Sport, 72, 335-344. doi: 10.1080/02701367.2001.10608970.

Wulf, G., \& Su, J. (2007). An external focus of attention enhances golf shot accuracy in beginners and experts. Research Quarterly for Exercise \& Sport, 78, 384-389. doi: 10.1080/02701367.2007.10599436.

Wulf, G., Töllner, T., \& Shea, C. H. (2007). Attentional focus effects as a function of task difficulty. Research Quarterly for Exercise \& Sport, 78, 257-264. doi: 10.1080/02701367.2007.10599423. 
2

3

4

5

6

7

8

9

10

11

12

13

14

15

16

17

18

19

20

21

22

23

24

25

26

27

28

29

30

31

32

33

34

35

36

37

38

39

40

41

42

43

44

45

46

47

48

49

50

51

52

53

54

55

56

57

58

59

60

\section{A.A. Author}

Figure 1. Median responses to the self-report manipulation check in each of the three attentional focus conditions.

Figure 2. Median putting performance (points) in each of the three attentional focus

conditions (a higher score indicates better performance). Median absolute deviations are represented on the figure by the error bars attached to each column. 


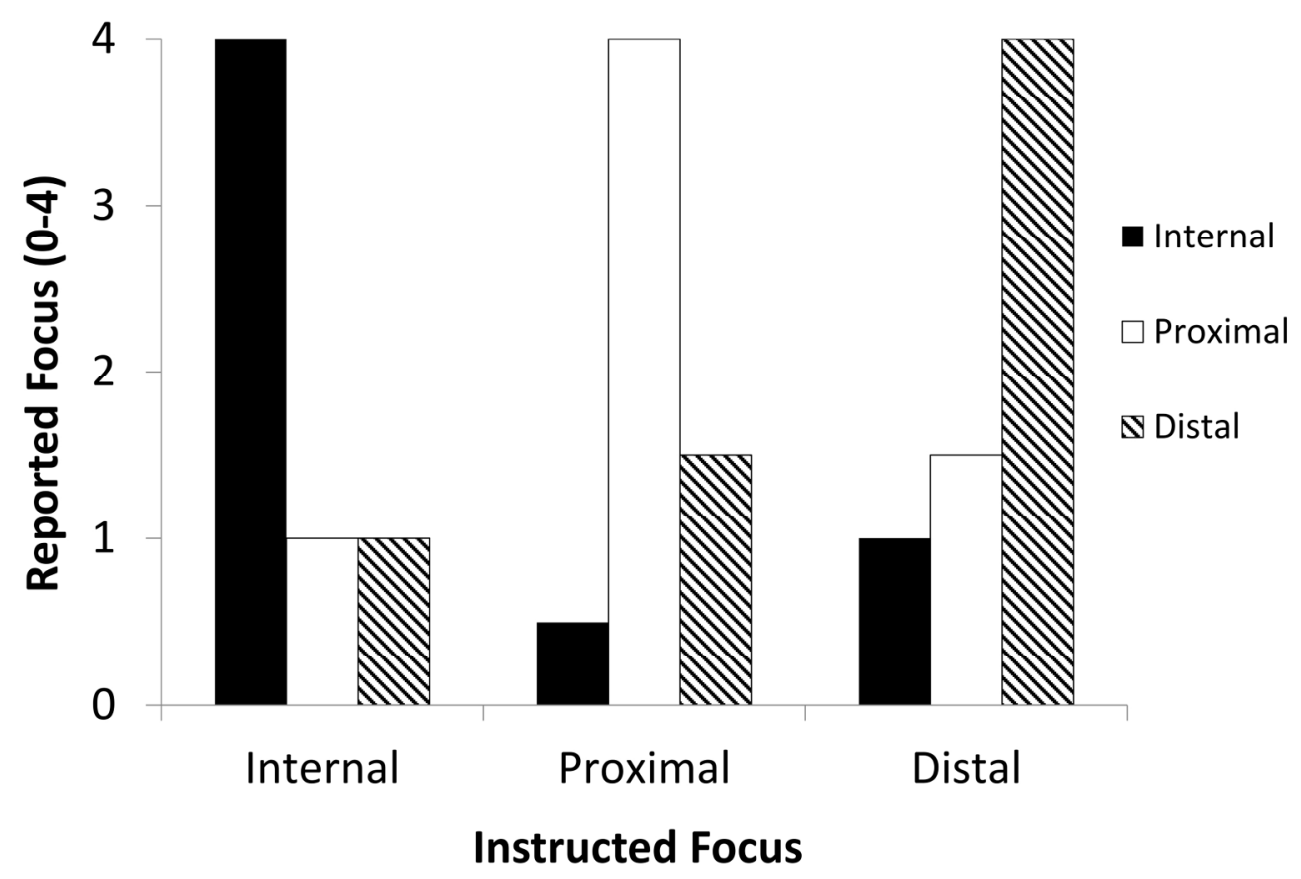

254x190mm (300 x 300 DPI)

34

35

36

37

38

39

40

41

42

43

44

45

46

47

48

49

50

51

52

53

54

55

56

57

58

59 


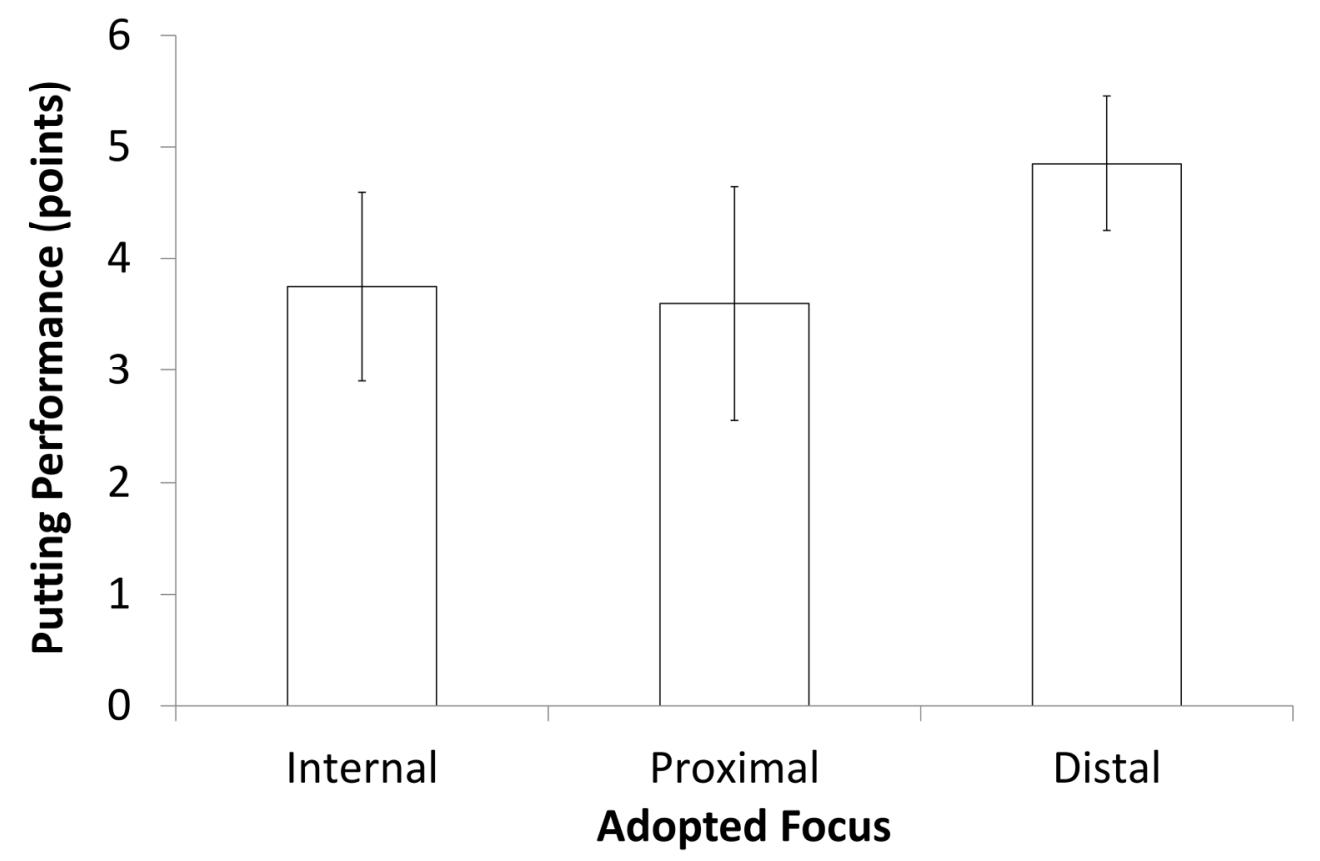

$254 \times 190 \mathrm{~mm}(300 \times 300 \mathrm{DPI})$

URL: http://mc.manuscriptcentral.com/rijs 